\title{
Phacoemulsification under topical anesthesia alone versus topical anesthesia with intracameral lodocaine injection
}

\author{
Dr Dipti N Kale ${ }^{1}$, Dr Yumnam Chingsuingamba ${ }^{2}$ \\ ${ }^{1,2}$ (Department of ophthalmology, Regional institute of medical sciences, Imphal)
}

\begin{abstract}
Purpose: goal was to study the intra-operative pain experienced by patients when topical alone was used compared with topical anesthesia with intracameral lidocaine injection. To study the patient and surgeon satisfaction when the two different types of anesthesia are used. Setting/Venue: Regional Institute of Medical Sciences, Imphal, India. Methods: 40 patients of cataract divided into two groups, A and B each containing 20 patients were included in the study. phacoemulsification was performed on group A under topical anesthesia with proparacaine hydrochloride $0.5 \%$ and on group B under topical anesthesia along with intracameral infiltration of $2 \%$ lidocaine. Un co-operative patients, those with previous ocular trauma and corneal opacities were excluded from the study. All patients in both groups were operated by the same surgeon. The surgeon and patient satisfaction score was entered in a standardized proforma. Results: 6 patients in group A felt no pain while 3 patients felt pain upto the extent that $0.5 \mathrm{cc}$ of $2 \%$ lidocaine was needed to infiltrate at the phaco port site. Thereafter the procedure was competed comfortably and pain free. Mean phacoemulsification time was 2.5 minutes and mean operation time was 30 minutes. In group $B$, the patients were operated after infiltration of 0.5 cc $2 \%$ lidocaine injection. 16 patients were operated pain free and 4 patients had mild pain. The mean phacoemulsification time was 2.2 minutes and the mean operation time was 30 minutes Conclusions: The intracameral infiltration of $2 \%$ lidocaine injection with topical anesthesia is superior to topical anesthesia with proparacaine hydrochloride alone during phacoemulsification ensuring patients and surgeons comfort. None of the patients in any group showed the complication as sometimes seen in periocular and retrobulbar anesthesia
\end{abstract}

Keywords: intracameral lidocaine, intraoperative pain, phacoemulsification, topical anesthesia, surgeon's satisfaction

\section{Introduction}

Advances in cataract surgery techniques with the advent of phacoemulsification and the consequent reduced surgical time, led to a renewed interest in topical anesthesia.

\section{Purpose Of Study}

Our goal was to study the intra-operative pain experienced by patients when topical alone was used compared with topical anesthesia with intracameral lidocaine injection. To study the patient and surgeon satisfaction when the two different types of anesthesia are used.

\section{Criteria For Selection Of Cases:}

\section{Patient Selection}

Cases of both sexes and those who fell in the age group of fifty years or above with significant cataract causing impairment of visual functions not correctable by glasses or with unacceptable glare, polyopia, or reduced quality of vision attributable to senile cataract and willing for cataract surgery were included in the study.

\section{Criteria For Exclusion Of Cases:}

1. Patients with types of cataract other than senile cataract

2. Patients who were unable to understand verbal command

3. Sensitivity to lidocaine

4. Patients with anxiety and with unintentional eye movement

5. Patients who did not give consent

\section{Anaesthesia Technique}

After approval for the study had been obtained from the ethics committee, written informed consent that included an explanation of the study design and goals were obtained from all patients. After having undergone a routine preoperative ophthalmic examination, the patients were divided into two groups of 20 each. No sedative systemic medications were given to the patients preoperatively or postoperatively. All patients received topical phenylephrine 5\% and tropicamide $1 \%$ every ten minutes for 30 minutes before surgery. 


\section{Administration of Anaesthesia:}

One group of patients received topical Anaesthesia only in the form of $0.5 \%$ proparacaine eye drops. The eye drops were instilled into the eye to be operated fifteen minutes prior to surgery. Instillation was done three times, at an interval of five minutes. The other group of patients received topical proparacaine before the surgery and intracameral injection of $1 \%$ preservative free lidocaine intraoperatively.

\section{Surgical Technique}

All surgical procedures were performed in the same institute by the same surgeon. No bridle rectus suture was used. Clear corneal incision was given which was very small (about $3.5 \mathrm{~mm}$ ). Continuous curvilinear capsulorrhexis (CCC) of 4-5 mm was done. After doing the hydroprocedure, the lens nucleus was phacoemulsified buy crater or trench divide and conquer technique. Cortical matter was aspirated out using two separate canulas (bimanual I/A). Viscoelastic material was injected into the capsular bag and in the anterior chamber before IOL implantation. The foldable IOLs used were mostly AcrysofIQ and AcryIOL. The foldable IOL was first mounted into the cartridge of the lens and then delivered into the capsular bag. The viscoelastic was then aspirated and anterior chamber was formed BSS. $0.5 \mathrm{cc}$ vigamox was injected intracamerally. The side port incisions are closed by edematising the cornea at the incision sites.

\section{Pain assessment:}

On completion of the operation, each patient was shown a visual analogue scale with numeric and descriptive ratings from 0 (no pain) to 10 (severe pain). Patients were asked to use this 10-point scale to rate the level of pain felt during the operation, including the pain felt after delivery of Anaesthesia. In addition, any verbal expression of pain that patients made during the operation (eg, on manipulation of iris) was recorded. Patients were asked to inform the surgeon if they experienced any pain at any point of the surgery. If so, additional anesthesia was given. Also, the level of patient satisfaction, the need for supplemental anaesthesia, level of ocular motility, surgical complications and postoperative visual acuity were recorded.

\section{Age Distribution:}

\section{Results And Observations}

The age distribution of the patients were as below which shows preponderance of the cases in the age group of 60 to 69 years, that is 18 .

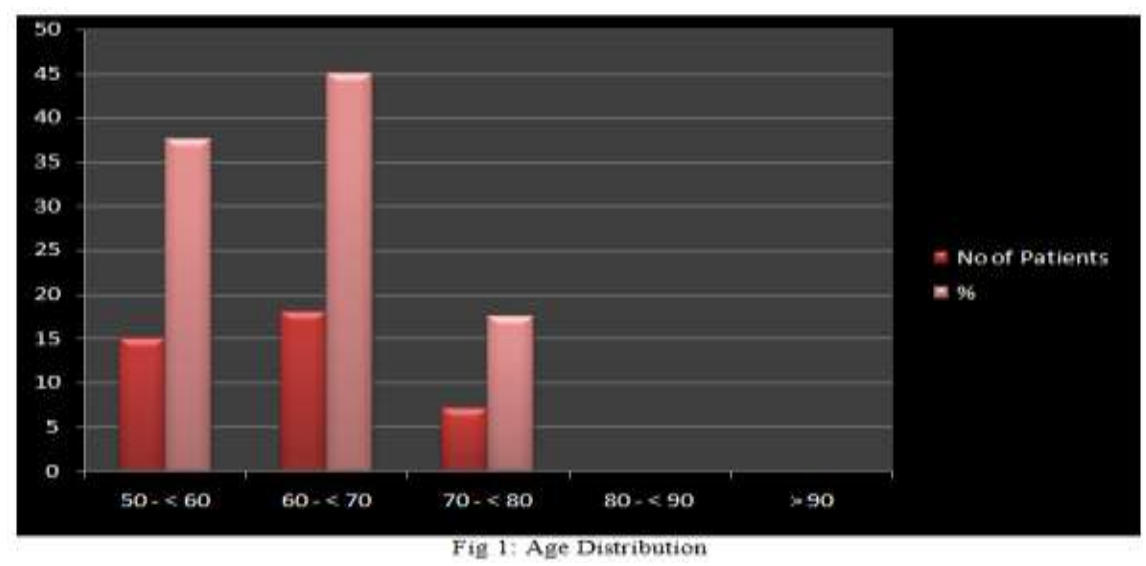

\section{Sex Distribution:}

Out of the 40 cases of this study, 26 were male and 14 were female.

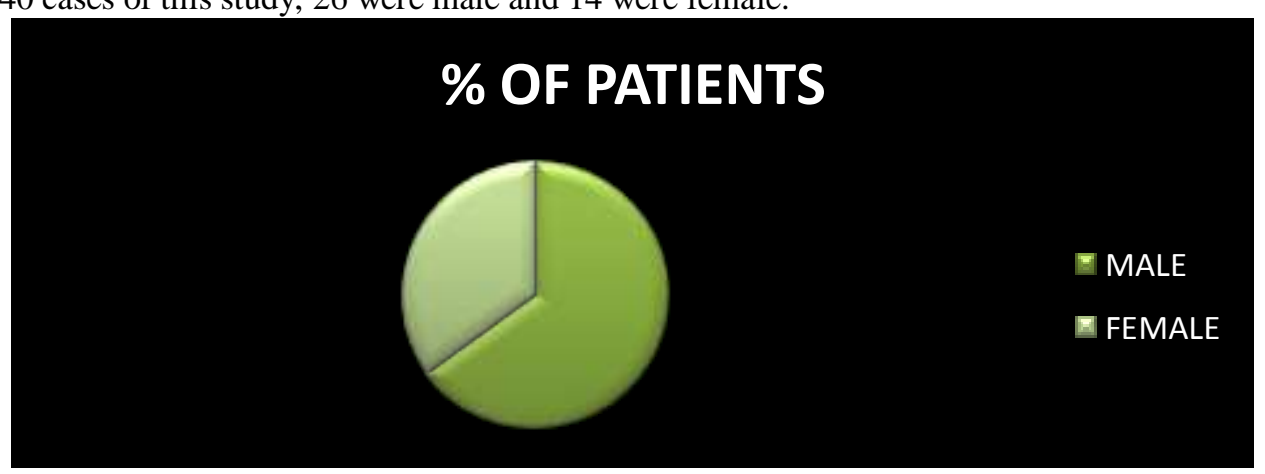

Fig 2: Sex Distribution 
Type of Cataract:

Of this study, 23 had cortical cataract, of which 13 were immature, 8 were mature and 2 were hypermature. Nuclear cataracts were encountered in 14 eyes of which 7 were immature, 6 were mature and 1 was hypermature.

\section{General condition of the patients:}

6 patients of this study were found to be diabetic of which 4 were males and 2 were females. All these patients were non insulin dependent diabetic with good control of blood sugar with oral hypoglycaemic agents. None of these patients had evidence of diabetic retinopathy. 4 cases of this study were documented as hypertensives that needed antihypertensive medications for regulation of blood pressure. All other patients of this study were in good physical and mental health for the age.

\section{Preoperative Visual Acuity Status:}

Among the 40 cases, 24 were planned for operation on the right eye and 16 on the left eye. The preoperative vision of these eyes was as below:-

\begin{tabular}{|c|c|c|}
\hline Vision & No. Of cases & \% of cases \\
\hline PL & 3 & 7.5 \\
\hline HM & 6 & 15 \\
\hline FC less than $3 \mathrm{~m}$ & 9 & 22.5 \\
\hline FC at $3 \mathrm{~m}(3 / 60)$ & 11 & 27.5 \\
\hline $6 / 60$ & 8 & 20 \\
\hline $6 / 36$ & 3 & 7.5 \\
\hline
\end{tabular}

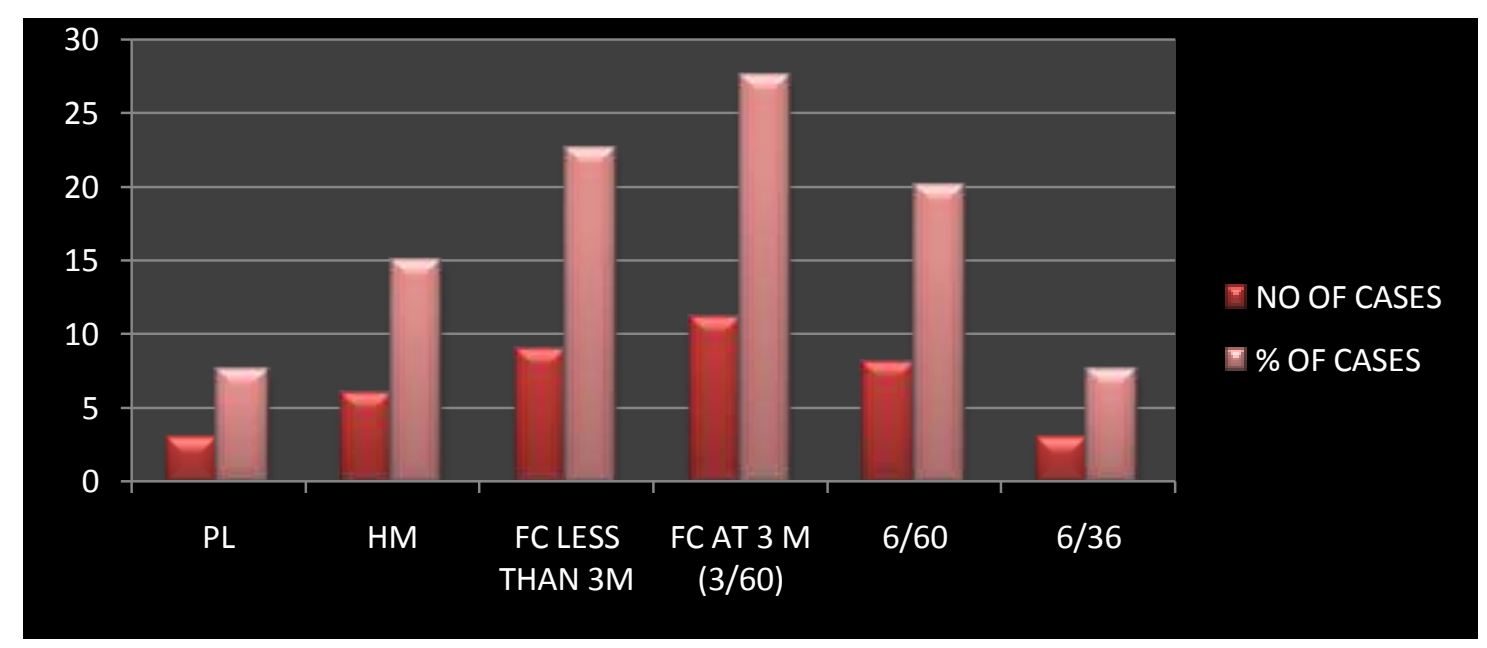

Intraoperative Pain:

Fig 3. Preoperative Visual Acuity

Out of the twenty patients who received only topical Anaesthesia, 6 patients experienced no pain, 10 patients experienced mild pain, 3 patients needed supplemental intracameral lidocaine injection. After intracameral lidocaine injection the procedure was uneventful. 1 patient experienced severe pain and had to be given peribulbar injection of anesthetic solution. Majority of the patients who experienced pain was during incision and capsulorrhexis. The pain scores as marked by the patients on the visual analogue scale were as follows : $0,0,0,0,0,0,1,2,3,3,3,2,2,2,3,1,4,5,6,4$.

Out of the twenty patients who received topical anaesthesia with intracameral lidocaine injection, 16 patients experienced no pain and 4 patients had mild pain. No patient had severe pain during the entire intervention. The pain scores as marked by the patients on the visual analogue scale were as follows: $0,0,0,0,0,0,0,0,0,0,0,0,0,0,0,0,3,2,2,1$.

The mean pain scores of the two groups were $2.05+/-1.82$ standard deviation and $0.40+/-0.88$ standard deviation for the patients who received topical anesthesia alone and those who received intra cameral lidocaine with topical anesthesia respectively.

Paired $t$ test was applied to calculate the significance. In conclusion the difference between the measures was found to be statistically significant $(\mathrm{P}<0.001)$. 


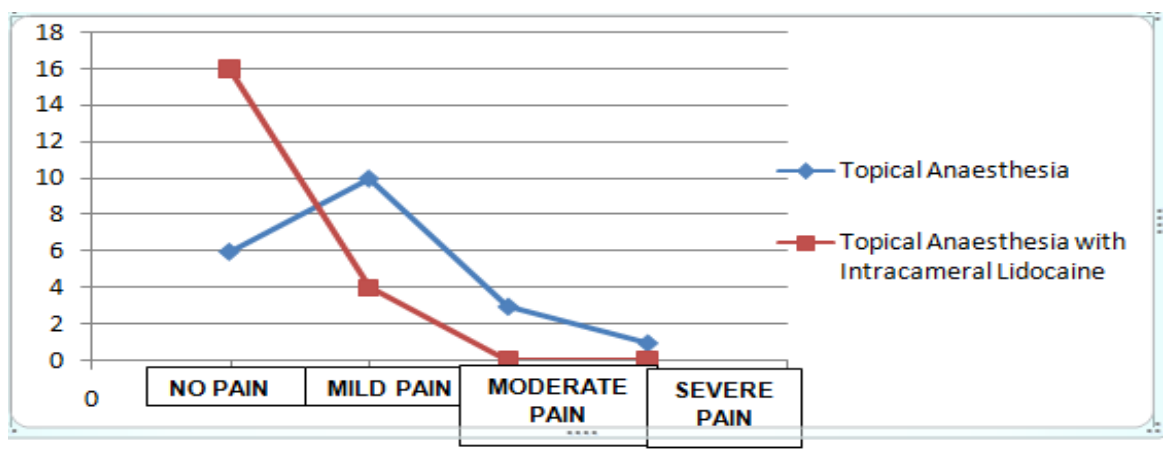

Fig 4. Intraoperative Pain

\section{Intraoperative Surgical Difficulties:}

Eye motility and a poor patient compliance were the main reasons for surgical difficulties. In patients who received only topical anaesthesia, disturbing eye movements were recorded in 2 patients. No eye movement and some eye movements were recorded in 13 and 5 patients respectively.

In patients who received intracameral lidocaine injection with topical anaesthesia, no movement was recorded in 18 patients and only some movements in 2 patients. Disturbing eye movement was not recorded in even a single patient.

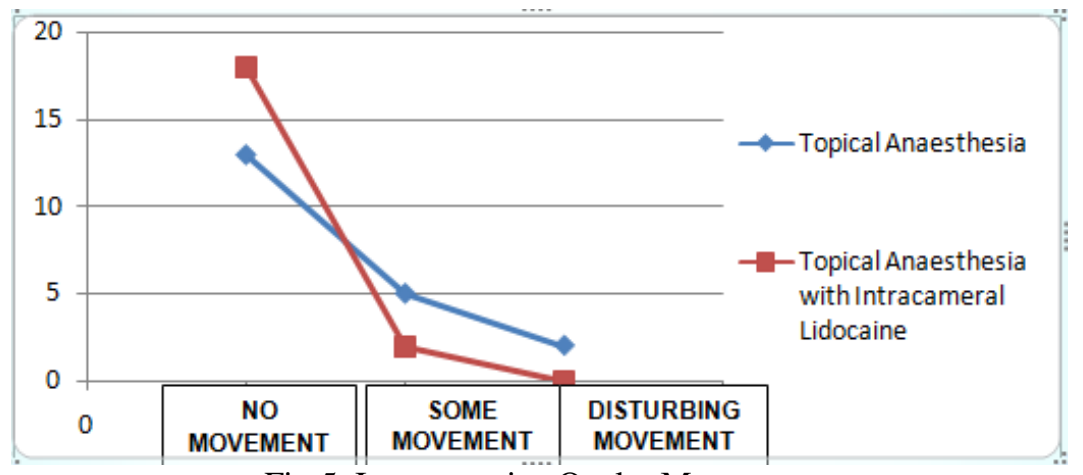

Fig 5. Intraoperative Ocular Movement

\section{Intra And Post Operative Complications:}

No serious intra operative or postoperative complications occurred.

Patient Satisfaction:

The following table shows the satisfaction pattern in patients who received topical anesthesia only. 14 patients who received topical anaesthesia were extremely satisfied. 4 patients were satisfied and 2 patients were dissatisfied. 17 patients who received topical anaesthesia with intracameral lidocaine were extremely satisfied, 3 were satisfied and no patient was dissatisfied.

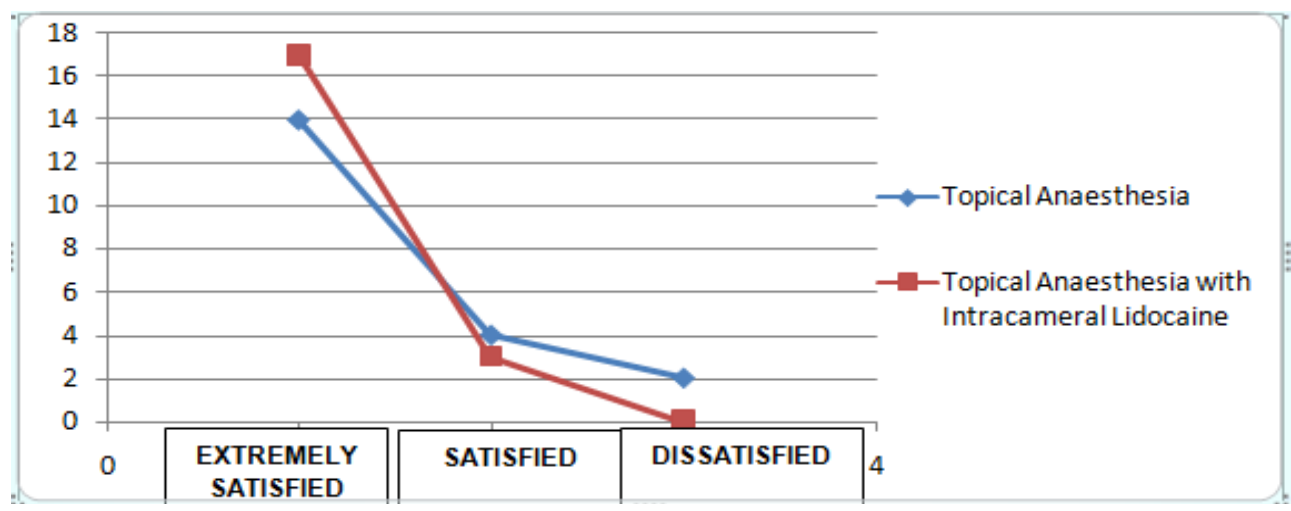

Fig 6. Patient Satisfaction

In the topical Anaesthesia group, 3 patients said they would opt for another type of Anaesthesia (peribulbar or with intracameral lidocaine injection) for the second eye, while only 1 patient from the other group would opt for another type of Anaesthesia (like peribulbar injection). 


\section{Post Operative Visual Acuity:}

The postoperative vision of the patients was as below

\begin{tabular}{|c|c|c|}
\hline Vision & No. Of patients & \% of patients \\
\hline $6 / 6$ & 7 & 17.5 \\
\hline $6 / 9$ & 16 & 40 \\
\hline $6 / 12$ & 13 & 32.5 \\
\hline $6 / 18$ & 4 & 10 \\
\hline
\end{tabular}

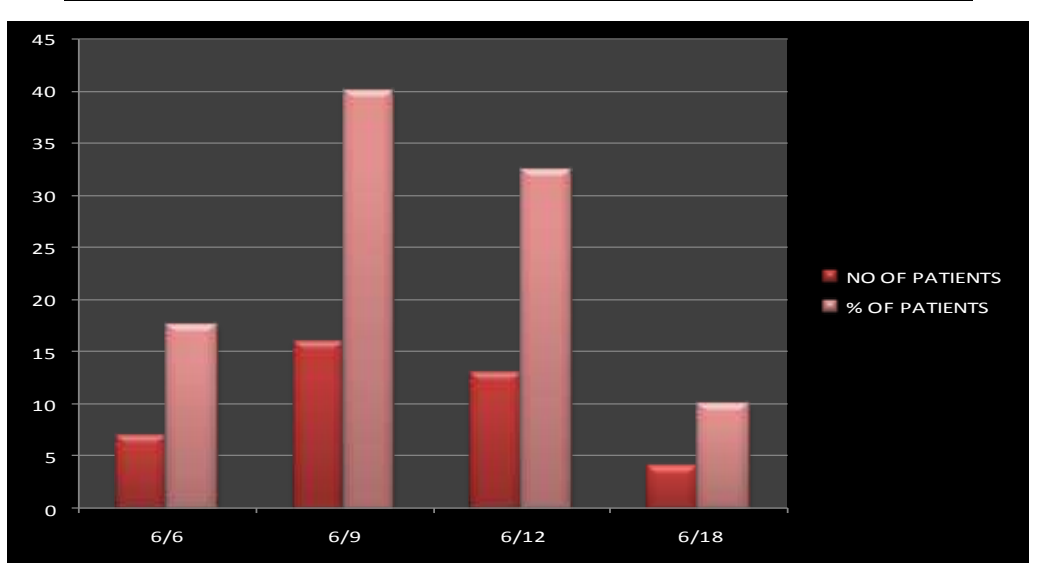

Although retrobulbar and peribulbar anaesthesia is sufficient for nerve blockage during cataract surgery complications related to the anaesthesia can be sight threatening or even life threatening. These complications can be prevented by the use of topical anaesthesia with or without intracameral lidocaine injection and the trend is to adopt this technique as a standard procedure. Sutureless clear corneal incision techniques have gained popularity among cataract surgeons owing to the many advantages they offer, including a reduction of ocular tissue manipulation and surgical time. With topical anaesthesia alone, potentially serious complications associated with retrobulbar and peribulbar Anaesthesia could be avoided. Since its introduction, topical anaesthesia has become increasingly popular.

On the other hand, topical anaesthesia can in certain circumstances be more demanding for the surgeon. The surgeon must be very competent and not every patient is a good candidate: that is the reason why very anxious patients, patients with communication problems, those with miotic pupils were excluded from our study. The main disadvantage of topical anaesthesia is the absence of akinesia. To acheive akinesia, our patients were asked to look at the light of the operating microscope. To reduce photophobia, light intensity of the microscope was set initially to its lowest level and gradually raised to normal values at the beginning of capsulorrhexis. With topical anaesthesia, only the trigeminal nerve endings in the cornea and the conjunctiva are blocked. Failure of topical anaesthesia to block the impulses in the long sensory fibers makes manipulation of the iris or stretching of the ciliary and zonular tissues uncomfortable for patients. As we know, some manipulations performed during phacoemulsification cause significant levels of pain.

O'Brien et al showed that hydrodissection, phacoemulsification, lens aspiration and intraocular lens insertion require the most manipulation of the iris and were associated with higher pain scores during surgery. Recently, a number of reports compared the patient comfort during cataract surgery with topical anaesthesia alone versus topical anaesthesia with intracameral lidocaine.

In a randomized controlled trial using a ten point visual analogue scale, Crandall and co-authors found no significant difference in intraoperative pain scores between patients who received topical anaesthesia alone or patients who received topical anaesthesia and intracameral lidocaine. However, other reports and our study have shown that patients under topical anaesthesia alone were more likely to experience pain and discomfort during iris manipulation and zonular stretching. We believe that for surgeons wanting to convert to topical anesthesia they have to be very comfortable with the phacoemulsification procedure so that it can be carried out rapidly and efficiently without much intraocular manipulations. In case the topical anesthesia is inadequate, the surgeon can fill the chamber with intracameral lidocaine injection

\section{Conclusion}

Surgery related pain and patient discomfort were significantly lesser in patients who received intra cameral lidocaine along with topical anesthesia as compared with patients who received topical anesthesia alone $(\mathrm{P}<0.001)$. Topical anesthesia with intracameral lidocaine injection is a justified means of improving patient comfort and co-operation of the patients in well selected cases undergoing phacoemulsification cataract surgery. 